\title{
HARDY INEQUALITIES AND CAFFARELLI-KOHN-NIRENBERG INEQUALITIES WITH RADIAL DERIVATIVE
}

\author{
Tuan Duy Nguyen, Le-Long Phi And WeiJia Yin
}

Abstract. In this paper, we study several inequalities of Hardy and Caffarelli-Kohn-Nirenberg type. We set up some optimal versions of these inequalities using the radial derivatives or the convex combinations of the full gradient and its radial part. We also exhibit their optimizers in some certain cases.

Mathematics subject classification (2010): 26D10, 35A23, 46E35.

Keywords and phrases: Hardy inequalities, Caffarelli-Kohn-Nirenberg inequalities, best constants, optimizers, radial derivative.

\section{REFERENCES}

[1] Adimurthi, Chaudhuri, N., Ramaswamy, M., An improved Hardy-Sobolev inequality and its application, Proc. Amer. Math. Soc. 130 (2002), no. 2, 489-505.

[2] AgueH, M., Sharp Gagliardo-Nirenberg inequalities via p-Laplacian type equations, NoDEA Nonlinear Differential Equations Appl. 15 (2008), no. 4-5, 457-472.

[3] Balinsky, A. A., Evans, W. D., Lewis, R. T., The analysis and geometry of Hardy's inequality, Universitext. Springer, Cham, 2015. xv+263 pp.

[4] Brezis, H., VÁzQUeZ, J. L., Blow-up solutions of some nonlinear elliptic problems, Rev. Mat. Univ. Complut. Madrid 10 (1997), no. 2, 443-469.

[5] Caffarelli, L., Kohn, R., Nirenberg, L., First order interpolation inequalities with weights, Compositio Math. 53 (1984), no. 3, 259-275.

[6] Caldiroli, P., Musina, R., Symmetry breaking of extremals for the Caffarelli-Kohn-Nirenberg inequalities in a non-Hilbertian setting, Milan J. Math. 81 (2013), no. 2, 421-430.

[7] Catrina, F., Costa, D. G., Sharp weighted-norm inequalities for functions with compact support in $\mathbb{R}^{N} \backslash\{0\}$, J. Differential Equations 246 (2009), no. 1, 164-182.

[8] CATrina, F., WAng, Z.-Q., On the Caffarelli-Kohn-Nirenberg inequalities: sharp constants, existence (and nonexistence), and symmetry of extremal functions, Comm. Pure Appl. Math. 54 (2001), no. 2, 229-258.

[9] Chanillo, A. L., Chanillo, S., MaAlaoui, A., Norm Constants in cases of the Caffarelli-KohnNirenberg inequality, Pacific J. Math. 292 (2018), no. 2, 293-303.

[10] Chiba, N., Horiuchi, T., Radial symmetry and its breaking in the Caffarelli-Kohn-Nirenberg type inequalities for $p=1$, Proc. Japan Acad. Ser. A Math. Sci. 92 (2016), no. 4, 51-55.

[11] Cianchi, A., Ferone, A., Hardy inequalities with non-standard remainder terms, Ann. Inst. H. Poincaré Anal. Non Linéaire 25 (2008), no. 5, 889-906.

[12] Costa, D. G., Some new and short proofs for a class of Caffarelli-Kohn-Nirenberg type inequalities, J. Math. Anal. Appl. 337 (2008), no. 1, 311-317.

[13] Davies, E. B., A review of Hardy inequalities, The Maz'ya anniversary collection, Vol. 2 (Rostock, 1998), 55-67, Oper. Theory Adv. Appl., 110, Birkhäuser, Basel, 1999.

[14] Del Pino, M., Dolbeault, J., Best constants for Gagliardo-Nirenberg inequalities and applications to nonlinear diffusions, J. Math. Pures Appl. (9) 81 (2002), no. 9, 847-875.

[15] Del-Pino, M., Dolbeault, J., The optimal Euclidean $L^{p}$-Sobolev logarithmic inequality, J. Funct. Anal. 197 (1) (2003), 151-161. 
[16] Dolbeault, J., Esteban, M. J., Loss, M., Rigidity versus symmetry breaking via nonlinear flows on cylinders and Euclidean spaces, Invent. Math. 206 (2016), no. 2, 397-440.

[17] Dong, M., LAm, N., Lu, G., Sharp weighted Trudinger-Moser and Caffarelli-Kohn-Nirenberg inequalities and their extremal functions, Nonlinear Anal. 173 (2018), 75-98.

[18] Evans, W. D., Lewis, R. T., Hardy and Rellich inequalities with remainders, J. Math. Inequal. 1 (2007), no. 4, 473-490.

[19] FilipPas, S., Tertikas, A., Optimizing improved Hardy inequalities, J. Funct. Anal. 192 (2002), no. 1, 186-233.

[20] Gazzola, F., Grunau, H.-C., Mitidieri, E., Hardy inequalities with optimal constants and remainder terms, Trans. Amer. Math. Soc. 356 (2004), no. 6, 2149-2168.

[21] Ghoussoub, N., Moradifam, A., Bessel pairs and optimal Hardy and Hardy-Rellich inequalities, Math. Ann. 349 (2011), no. 1, 1-57.

[22] Ghoussoub, N., Moradifam, A., Functional inequalities: new perspectives and new applications, Mathematical Surveys and Monographs, 187. American Mathematical Society, Providence, RI, 2013. xxiv+299.

[23] Ghoussoub, N., Moradifam, A., On the best possible remaining term in the Hardy inequality, Proc. Natl. Acad. Sci. USA 105 (2008), no. 37, 13746-13751.

[24] Gurka, P., Generalized Hardy's inequality, Časopis Pěst. Mat. 109 (1984), no. 2, 194-203.

[25] Ioku, N., Ishiwata, M., Ozawa, T., Sharp remainder of a critical Hardy inequality, Arch. Math. (Basel) 106 (2016), no. 1, 65-71.

[26] Kufner, A., Maligranda, L., Persson, L.-E., The Hardy Inequality. About its History and Some Related Results, Vydavatelský Servis, Pilsen, 2007.

[27] Kufner, A., Maligranda, L., Persson, L-E., The prehistory of the Hardy inequality, American Mathematical Monthly 113(8) (2006) 715-732.

[28] Kufner, A., Persson, L.-E., Samko, N., Weighted inequalities of Hardy type, Second edition, World Scientific Publishing Co. Pte. Ltd., Hackensack, NJ, 2017. xx+459 pp.

[29] LAm, N., A note on Hardy inequalities on homogeneous groups, Potential Anal. 51 (2019), no. 3, 425-435.

[30] LAm, N., Hardy and Hardy-Rellich type inequalities with Bessel pairs, Ann. Acad. Sci. Fenn. Math. 43 (2018), 211-223.

[31] Lam, N., General sharp weighted Caffarelli-Kohn-Nirenberg inequalities, Proc. Roy. Soc. Edinburgh Sect. A 149 (2019), no. 3, 691-718.

[32] Lam, N., Sharp weighted isoperimetric and Caffarelli-Kohn-Nirenberg inequalities, Adv. Calc. Var. DOI: https://doi.org/10.1515/acv-2017-0015.

[33] LAm, N., Lu, G., Sharp constants and optimizers for a class of the Caffarelli-Kohn-Nirenberg inequalities, Adv. Nonlinear Stud. 17 (2017), no. 3, 457-480.

[34] LAm, N., Lu, G., ZHANG, L., Factorizations and Hardy's type identities and inequalities on upper half spaces, Calc. Var. Partial Differential Equations 58 (2019), no. 6, Paper No. 183, 31 pp.

[35] LU, G., YANG, Q., Green's functions of Paneitz and GJMS operators on hyperbolic spaces and sharp Hardy-Sobolev-Maz'ya inequalities on half spaces, https://arxiv.org/abs/1903.10365.

[36] Lu, G., YAnG, Q., Paneitz operators on hyperbolic spaces and high order Hardy-Sobolev-Maz'ya inequalities on half spaces, Amer. J. Math. 141 (2019), no. 6, 1777-1816.

[37] Machihara, S., OZAWa, T., Wadade, H., Remarks on the Hardy type inequalities with remainder terms in the framework of equalities, In Asymptotic Analysis for Nonlinear Dispersive and Wave Equations, pp. 247-258. Mathematical Society of Japan, 2019.

[38] Masmoudi, N., About the Hardy Inequality, An Invitation to Mathematics: From Competitions to Research, edited by Dierk Schleicher, Malte Lackmann, Springer (2011) 165-180.

[39] Nguyen, T. D., Lam-Hoang, N., Nguyen, A. T., Hardy-Rellich identities with Bessel pairs, Arch. Math. (Basel) 113 (2019), no. 1, 95-112.

[40] Nguyen, T. D., Lam-Hoang, N., Nguyen, A. T., Hardy and Rellich inequalities with exact missing terms on homogeneous groups, J. Math. Soc. Japan 71 (2019), no. 4, 1243-1256.

[41] Nguyen, T. D., Lam-Hoang, N., Nguyen, A. T., Improved Hardy and Hardy-Rellich type inequalities with Bessel pairs via factorizations, J. Spectr. Theory, to appear.

[42] OpIC, B., Kufner, A., Hardy-type inequalities, Pitman Research Notes in Mathematics Series, 219. Longman Scientific \& Technical, Harlow, 1990. xii+333 pp. 
[43] Ruzhansky, M., Suragan, D., Hardy inequalities on homogeneous groups: 100 years of Hardy inequalities, Progress in Mathematics, Vol. 327, Birkhauser, 2019. xvi+588pp.

[44] Ruzhansky, M., Suragan, D., Yessirkegenov, N., Extended Caffarelli-Kohn-Nirenberg inequalities, and remainders, stability and superweights for Lp-weighted Hardy inequalities, Trans. Amer. Math. Soc. Ser. B, 5 (2018), 32-62.

[45] Sano, M., Takahashi, F., Scale invariance structures of the critical and the subcritical Hardy inequalities and their improvements, Calc. Var. Partial Differential Equations 56 (2017), no. 3, Art. 69 , $14 \mathrm{pp}$.

[46] ZHONG, X., Zou, W., Existence of extremal functions for a family of Caffarelli-Kohn-Nirenberg inequalities, https://arxiv.org/abs/1504.00433 